\title{
Incoherent bandpass spatial filtering with longitudinal periodicity
}

\author{
Ting-Chung Poon and Guy Indebetouw
}

\begin{abstract}
We explore the possibility of realizing bandpass filtering with longitudinal periodicity in incoherent systems. The necessary condition for spatial filtering to be longitudinally periodic is derived. Results indicate that bandpass filtering with longitudinal periodicity can be achieved in a two-pupil system with Fresnel zone plates with a small opening ratio as the pupils.
\end{abstract}

\section{Introduction}

The phenomenon of wave field replication without imaging elements is commonly known as the self-imaging effect. This effect has been observed and studied extensively ${ }^{1-5}$ and found applications in areas such as interferometry, ${ }^{6,7}$ spatial filtering, ${ }^{8,9}$ and acoustooptics. $^{10}$ We explore the possibility of achieving spatial filtering with longitudinal periodicity in incoherent systems. By filtering with longitudinal periodicity we mean that a certain spatial filtering operation in the transverse directions $(x, y)$ is repeated along the longitudinal direction $(z)$ at a periodic distance. Such systems may find applications in 3-D information processing, coding, structured illumination, and in 3-D space variant filtering. Specifically, the aspect of incoherent bandpass filtering is emphasized in this paper. Optical transfer functions (OTFs) with bandpass characteristics cannot be synthesized in conventional single pupil systems. One, therefore, needs to use the method of pupil function replication ${ }^{11}$ or employ twopupil systems. ${ }^{12-16}$ In Sec. II we develop the mathematical formalism used to describe the response of a defocused two-pupil system. The condition under which the OTF is longitudinally periodic is derived in Sec. III. Section IV describes how a Fresnel zone plate (FZP) can act as a self-imaging pupil. In Sec. V, we consolidate the results of Sec. III and IV to investigate bandpass filtering with longitudinal periodicity in a two-pupil system. Calculated results are obtained

Both authors are with Virginia Polytechnic Institute \& State University, Blacksburg, Virginia 24061-0111; T. C. Poon is with the Bradley Department of Electrical Engineering, Image Processing Laboratory, and G. Indebetouw is with the Physics Department.

Received 4 December 1989.

0003-6935/90/233345-07\$02.00/0.

(C) 1990 Optical Society of America. and shown to be consistent with theoretical predictions. Finally, in Sec. VI, are some concluding remarks.

\section{Defocused OTF of Two-Pupil Systems}

It is well known that the defocused OTF of an incoherent imaging system can be expressed as the autocorrelation of a defocused pupil function ${ }^{17}$ :

$$
\begin{aligned}
\operatorname{OTF}(\bar{\rho}, z) & =P(\bar{\rho}, z) \star P(\bar{\rho}, z) \\
& =\int P\left(\overline{\rho^{\prime}}-\bar{\rho}\right) P^{\star}\left(\overline{\rho^{\prime}}\right) \exp \left[j \pi \lambda z\left(\left|\bar{\rho}^{\prime}-\bar{\rho}\right|^{2}-{\rho^{\prime}}^{2}\right)\right] d^{2} \rho^{\prime} .
\end{aligned}
$$

Here $P(\bar{\rho}, z)$ is the defocused pupil function given by

$$
P(\bar{\rho}, z)=p(\bar{\rho}) \exp \left(j \pi \lambda z \rho^{2}\right),
$$

where $p(\bar{\rho})$ is the in-focus pupil function, $\lambda$ is the wavelength, and $z$ is the defocused distance measured away from the focal plane of the second lens in Fig. 1.

For a given aperture function $A(\bar{r})$ located in the $\bar{r}$ plane as shown in Fig. 1, the corresponding defocused pupil, expressed in terms of the spatial frequency $\bar{\rho}=$ $\vec{r} / \lambda f$, for a focal length $f$ and in the paraxial approximation is given by

$$
P(\bar{\rho}, z)=A(\lambda f \bar{\rho}) \exp \left(j \pi \lambda z \rho^{2}\right) .
$$

For a two-pupil system ${ }^{12,13}$ the defocused pupil is

$$
P(\bar{\rho}, z)=U(\bar{\rho}, z)+V(\bar{\rho}, z),
$$

where $U(\bar{\rho}, z)=u(\bar{\rho}) \exp \left(j \pi \lambda z \rho^{2}\right)$ and $V(\bar{\rho}, z)=v(\bar{\rho})$ $\exp \left(j \pi \lambda z \rho^{2}\right)$. Thus the corresponding defocused OTF of the two-pupil system becomes, using Eq. (1),

$$
\mathrm{OTF}=U \star U+V \star V+U \star V+V \star U .
$$

Note that the autocorrelation terms are always of low pass characteristics. To achieve spatial filterings with properties other than low pass, the cross-terms need to be extracted. The cross-correlation of the interactive terms can be separated from the autocorrelation (noninteractive) terms by the use of a spatial frequency offset $^{18-20}$ or a temporal frequency offset. ${ }^{21}$ A simple 
pupil interaction processing technique, in which both spatial carrier and temporal carrier offset are brought about by acoustooptics, has also been described. ${ }^{22}$ In the acoustooptic two-pupil interactive systems, the defocused OTF is the cross-correlation of the two defocused pupils. ${ }^{23}$ Using Eqs. (1) and (4), we have

$$
\begin{aligned}
\operatorname{OTF}(\bar{\rho}, z) & =U \star V \\
& =\int u\left(\bar{\rho}^{\prime}-\bar{\rho}\right) v^{\star}\left(\overline{\rho^{\prime}}\right) \exp \left[j \pi \lambda z\left(\left|\bar{\rho}^{\prime}-\bar{\rho}\right|^{2}-{\rho^{\prime}}^{\prime 2}\right)\right] d^{2} \rho^{\prime} .
\end{aligned}
$$

A real time system based on the acoustooptic approach has been described for realizing simultaneously a low pass filter and a first- and second-order differentiation. ${ }^{24}$ A similar system has been used recently for textural edge extraction. ${ }^{25}$

\section{Condition for Longitudinally Periodic OTF}

$\operatorname{An} \operatorname{OTF}(\bar{\rho}, z)$, which is periodic in $z$ with a period $z_{0}$, can be expressed in terms of a Fourier series expansion:

$$
\operatorname{OTF}(\bar{\rho}, z)=\sum_{l} a_{l}(\bar{\rho}) \exp \left(j 2 \pi l z / z_{0}\right) .
$$

By equating Eqs. (7) and (6), we can establish the requirement for $u(\bar{\rho})$ and $v(\bar{\rho})$ so that the two pupils produce a spatial filtering function that repeats longitudinally at regular intervals $z_{0}$ :

$$
\begin{aligned}
\int u\left(\bar{\rho}^{\prime}-\bar{\rho}\right) v^{\star}\left(\overline{\rho^{\prime}}\right) \exp \left[j \pi \lambda z\left(\left|\bar{\rho}^{\prime}-\bar{\rho}\right|^{2}-\rho^{\prime 2}\right)\right] d^{2} \rho^{\prime} \\
=\sum_{l} a_{l}(\bar{\rho}) \exp \left(j 2 \pi l z / z_{0}\right) .
\end{aligned}
$$

To have the same functional dependence on $z$ on both sides of Eq. (8), $\left|\bar{\rho}^{\prime}-\bar{\rho}\right|$ and $\rho^{\prime}$ must take on discrete values. Thus $u(\bar{\rho})$ and $v(\bar{\rho})$ are nonzero only on discrete rings, and the two pupils must thus take the following form:

$$
\begin{aligned}
& u(\bar{\rho})=\sum_{n} \mu_{n}(\bar{\rho}) \delta\left(\bar{\rho}-\rho_{n}\right), \\
& v(\bar{\rho})=\sum_{m} \nu_{m}(\bar{\rho}) \delta\left(\bar{\rho}-\rho_{m}\right) .
\end{aligned}
$$

Substituting Eq. (9) into Eq. (8), the OTF can then be written as

$$
\begin{aligned}
& \operatorname{OTF}(\bar{\rho}, z)=\int \sum_{n} \sum_{m} \mu_{n} \delta\left(\left|\bar{\rho}^{\prime}-\bar{\rho}\right|-\rho_{n}\right) \nu_{m} \delta\left(\rho^{\prime}-\rho_{m}\right) \\
& \times \exp \left[j \pi \lambda z\left(\rho_{n}^{2}-\rho_{m}^{2}\right)\right] d^{2} \rho^{\prime}=\sum_{n} \sum_{m} \mu_{n} \nu_{m} C\left(\rho_{n}, \rho_{m}, \bar{\rho}\right) \\
& \times \exp \left[j \pi \lambda z\left(\rho_{n}^{2}-\rho_{m}^{2}\right)\right],
\end{aligned}
$$

where $C\left(\rho_{n}, \rho_{m}, \bar{\rho}\right)$ is the intersection of the two rings $\delta\left(\left|\bar{\rho}^{\prime}-\bar{\rho}\right|-\rho_{n}\right)$ and $\delta\left(\rho^{\prime}-\rho_{m}\right)$. The situation is illustrated in Fig. 2.

Now, comparing the exponentials of Eqs. (10) and (7), it is evident that $\rho_{n}^{2}-\rho_{m}^{2}$ must be an integer. Thus we have

$$
\begin{aligned}
& \rho_{n}^{2}=n \rho_{1}^{2}, \\
& \rho_{m}^{2}=m \rho_{1}^{2},
\end{aligned}
$$

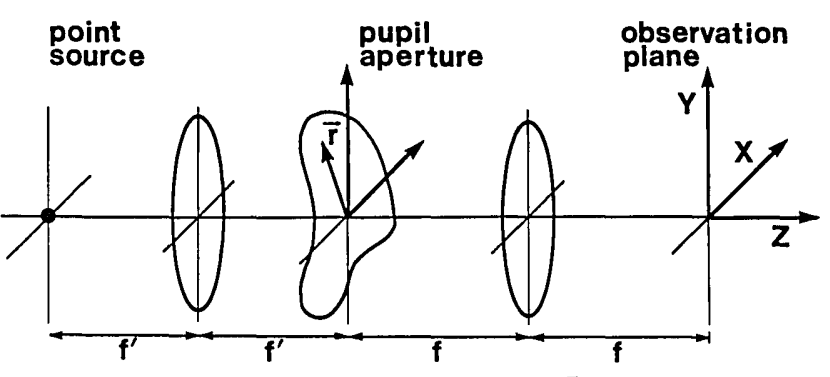

Fig. 1. Optical system defining the aperture $\bar{r}$-plane and the defocused distance $(z)$.

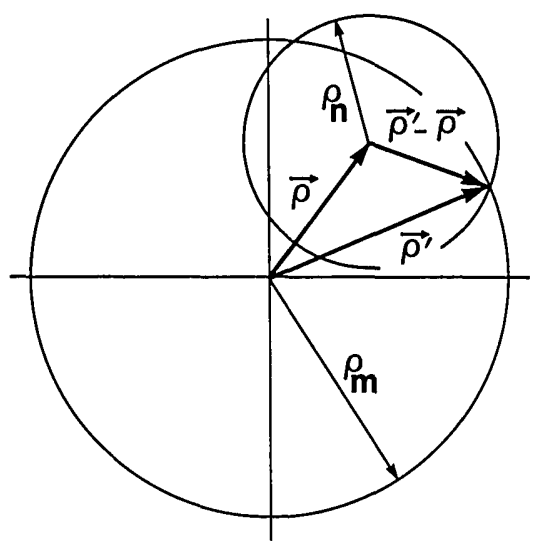

Fig. 2. Geometry illustrating the interactions of the two $\delta$-rings in the $\rho^{\prime}$-plane.

where $n$ and $m$ are some integers and $\rho_{1}$ is some constant. With condition (11), the required pupil functions which give rise to an OTF periodic in $z$ are of the form

$$
\begin{aligned}
& u(\bar{\rho})=\sum_{n} \mu_{n}(\bar{\rho}) \delta\left(\bar{\rho}-\sqrt{n} \rho_{1}\right), \\
& v(\bar{\rho})=\sum_{n} \nu_{m}(\bar{\rho}) \delta\left(\bar{\rho}-\sqrt{m} \rho_{1}\right) .
\end{aligned}
$$

This means that the two pupils must have a domain limited to rings with radii $\sqrt{n} \rho_{1}$. It is noteworthy to indicate that this condition is the same as that found by Montgomery for a pupil having self-imaging properties. $^{3}$

With condition (11), Eq. (10) can finally be written as

$$
\operatorname{OTF}(\bar{\rho}, z)=\sum_{n} \sum_{m} \mu_{n} \nu_{m} C_{n, m}(\bar{\rho}) \exp \left[j \pi \lambda z(n-m) \rho_{1}^{2}\right],
$$

where $C_{n, m}(\bar{\rho})=C\left(\rho_{n}, \rho_{m}, \bar{\rho}\right)$. Letting $n-m=l$ in Eq. (13) and comparing Eq. (13) with Eq. (7), we find that

$$
\begin{aligned}
& a_{l}(\bar{\rho})=\sum_{n} \mu_{n} \nu_{n-l} C_{n, n-l}(\bar{\rho}), \\
& z_{0}=\frac{2}{\lambda \rho_{1}^{2}} .
\end{aligned}
$$


The OTF characteristics are determined by the coefficients $\mu_{n}$ and $\nu_{m}$ which, in general, may be functions of an azimuthal angle $\theta$ as well as by the intersection $C_{n, m}$ of the rings. The periodic distance $z_{0}$ is wavelength dependent and a function of the radius $\rho_{1}$ of the first rings.

\section{Fresnel Zone Plate as a Self-Imaging Pupil}

The ring pupils found in Eq. (12) are Fresnel zone plate (FZP) apertures with a vanishingly small opening ratio. A practical realization of such apertures will obviously have a finite opening ratio. Such an unbounded FZP aperture has a transmission function given by

$$
A(\bar{r})=\sum_{n} A_{n} \exp \left(j 2 \pi n \frac{r^{2}}{R_{1}^{2}}\right),
$$

where $R_{1}$ is the radius of the first zone, and

$$
A_{n}=\frac{\sin \alpha n \pi}{n \pi}
$$

for a FZP having an opening ratio $\alpha$. Note that here the coefficients $A_{n}$ are constants, while in the general expression (7) for the $z$-periodic OTF, the coefficients $a_{l}$ can vary with an azimuthal angle $\theta$. If this FZP, placed in the $\bar{r}$-plane in Fig. 1, is illuminated by a point source located in the front focal plane of the first lens, multiple foci are observed along the longitudinal $z$ direction. To find the distance between the foci, we first express Eq. (15) as a defocused pupil. On substituting Eq. (15) into Eq. (3), we obtain

$$
P(\bar{\rho}, z)=\sum_{n} A_{n} \exp \left(j 2 \pi n \frac{\lambda^{2} f^{2}}{R_{1}^{2}} \rho^{2}\right) \exp \left(j \pi \lambda z \rho^{2}\right) .
$$

The amplitude of the point spread function (PSF) along the $z$-axis can then be found by evaluating the Fourier transform of Eq. (16) at $x=y=0$, giving

$$
\begin{aligned}
h(z) & =\int_{0}^{\infty} \sum_{n} A_{n} \exp \left[j 2 \pi\left(\frac{n \lambda^{2} f^{2}}{R_{1}^{2}}+\frac{\lambda z}{2}\right) \rho^{2}\right] 2 \pi \rho d \rho \\
& =\sum A_{n}^{\prime} \delta\left(z+n \frac{2 \lambda f^{2}}{R_{1}^{2}}\right),
\end{aligned}
$$

where $A_{n}^{\prime}$ has absorbed all constants resulting from evaluation of the integral. With a FZP of finite size, the $\delta$-functions are convolved with a smoothing function, the width of which is inversely proportional to the radius of the outermost zone. From Eq. (17), we see that the point source is imaged at locations given by

$$
z_{n}=n \frac{2 \lambda f^{2}}{R_{1}^{2}} .
$$

These are the positions at which the self-images appear. The distance $z_{0}$ between the self-images is $2 \lambda f^{2} / R_{1}^{2}$. Note that the intensity at $z_{n}$ is proportional to $\left(A_{n}^{\prime}\right)^{2}$. To obtain equal intensity at these locations, the $A_{n}$ terms should be independent of $n$. This occurs if the opening ratio of the zones approaches zero be- cause $A_{n} \rightarrow \alpha$ as $\alpha \rightarrow 0$. Therefore, a thin FZP can be used as an approximate self-imaging pupil.

\section{Bandpass Filtering with Longitudinal Periodicity}

To achieve bandpass filtering, the two pupils must be distinct so that their cross correlation produces the desired spatial frequency response. In particular, for a bandpass filter, the dc should not be transmitted. This means that the overlap area of the two apertures should vanish. To ensure filtering with longitudinal periodicity, the pupils must also be of the self-imaging type. Hence bandpass filtering with longitudinal periodicity can be accomplished by employing, for example, the following apodized FZP pupils in a two-pupil interaction system:

$$
\begin{aligned}
& u(\bar{\rho})=\exp \left(-\frac{\rho^{2} \lambda^{2} f^{2}}{R_{1}^{2}} \frac{1}{N}\right)\left[\sum_{n} A_{n} \exp \left(j 2 \pi n \frac{\lambda^{2} f^{2}}{R_{1}^{2}} \rho^{2}\right)\right], \\
& v(\bar{\rho})=\exp \left(-\frac{\rho^{2} \lambda^{2} f^{2}}{R_{1}^{2}} \frac{1}{N}\right)\left[\sum_{m}(-1)^{m} A_{m} \exp \left(j 2 \pi m \frac{\lambda^{2} f^{2}}{R_{1}^{2}} \rho^{2}\right)\right]
\end{aligned}
$$

where

$$
A_{n}=\frac{\sin \alpha n \pi}{n \pi} \text { and } A_{m}=\frac{\sin \beta m \pi}{m \pi} \text {. }
$$

The terms $u(\bar{\rho})$ and $v(\bar{\rho})$ are two FZPs with Gaussian apodizations. The apodization is introduced to limit the number $N$ of zones in the pupil while still leading to an analytical solution for the OTF. In addition, this apodization may be thought of as representing the effect of a Gaussian light beam illumination. The opening ratios $\alpha$ and $\beta$ in EQs. (19) are $<0.5$ to ensure that the two pupils have no common area of clear aperture. Figure 3 shows the unapodized FZPs. To obviate the specification of specific dimensions, we introduce the following nomalized coordinates:

$$
\hat{\rho}=\frac{\rho \lambda f}{R_{1}}, \xi=\frac{z}{z_{0}},
$$

where $z_{0}=2 \lambda f^{2} / R_{1}^{2}$ is the distance between the foci. Substituting Eq. (19) into Eq. (6) and using the normalization parameters in Eqs. (20), we find

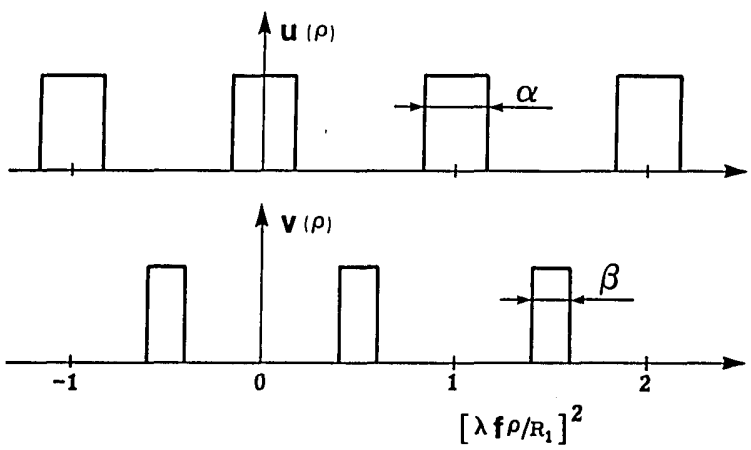

Fig. 3. Fresnel zone plate apertures plotted against the normalized coordinate $\hat{\rho}^{2}=\left(\rho \lambda f / R_{1}\right)^{2}$ used to approximate a longitudinally periodic bandpass filter. 

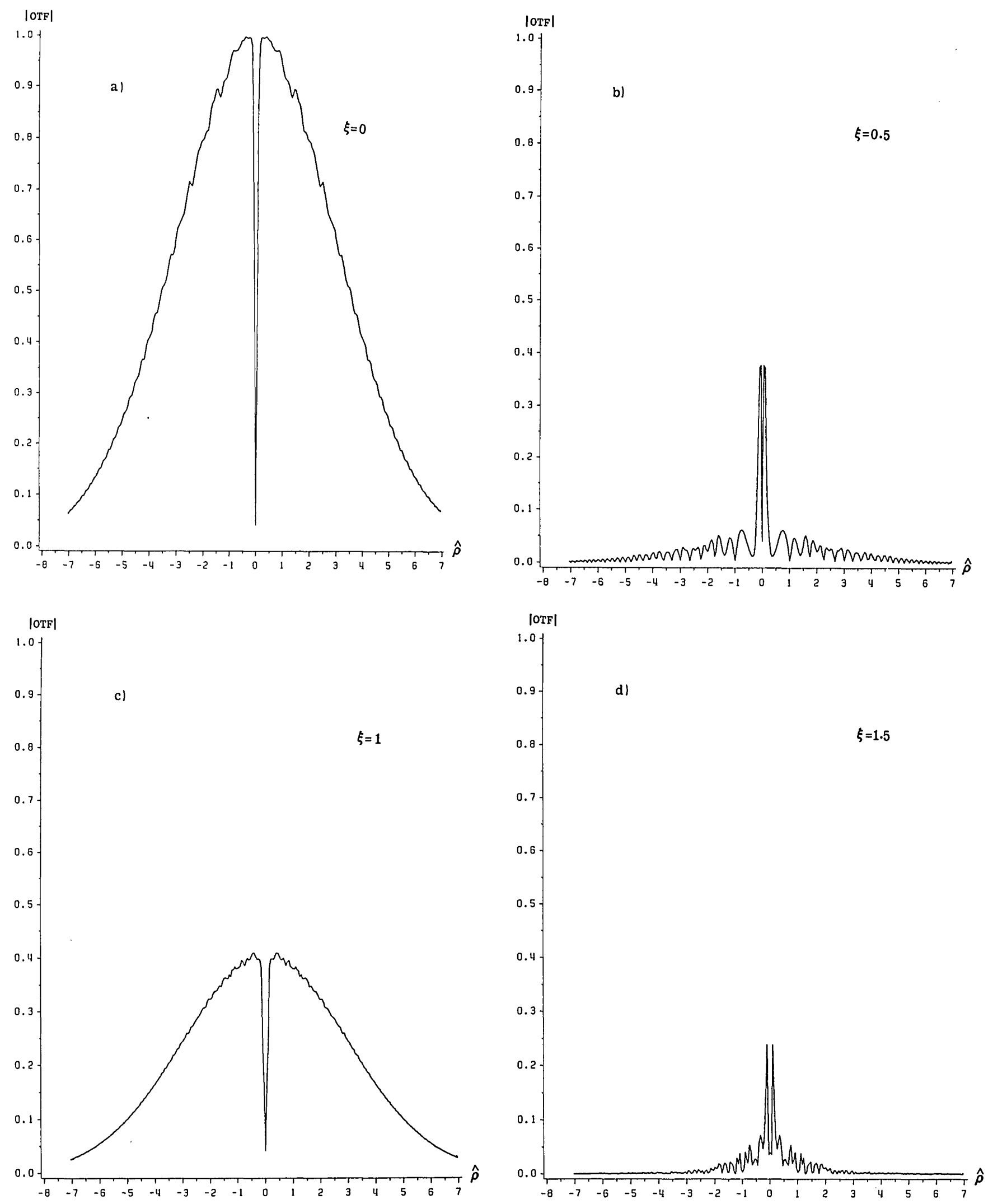

Fig. 4. $|\mathrm{OTF}|$ vs $\hat{\rho}$ as a function of $\xi$, the normalized defocused distance. The opening ratios of the FZPs are $\alpha=\beta=0.5$ and $N=9$ : (a) $\xi=0$, (b) $\xi=0.5$, (c) $\xi=1$, (d) $\xi=1.5$ 

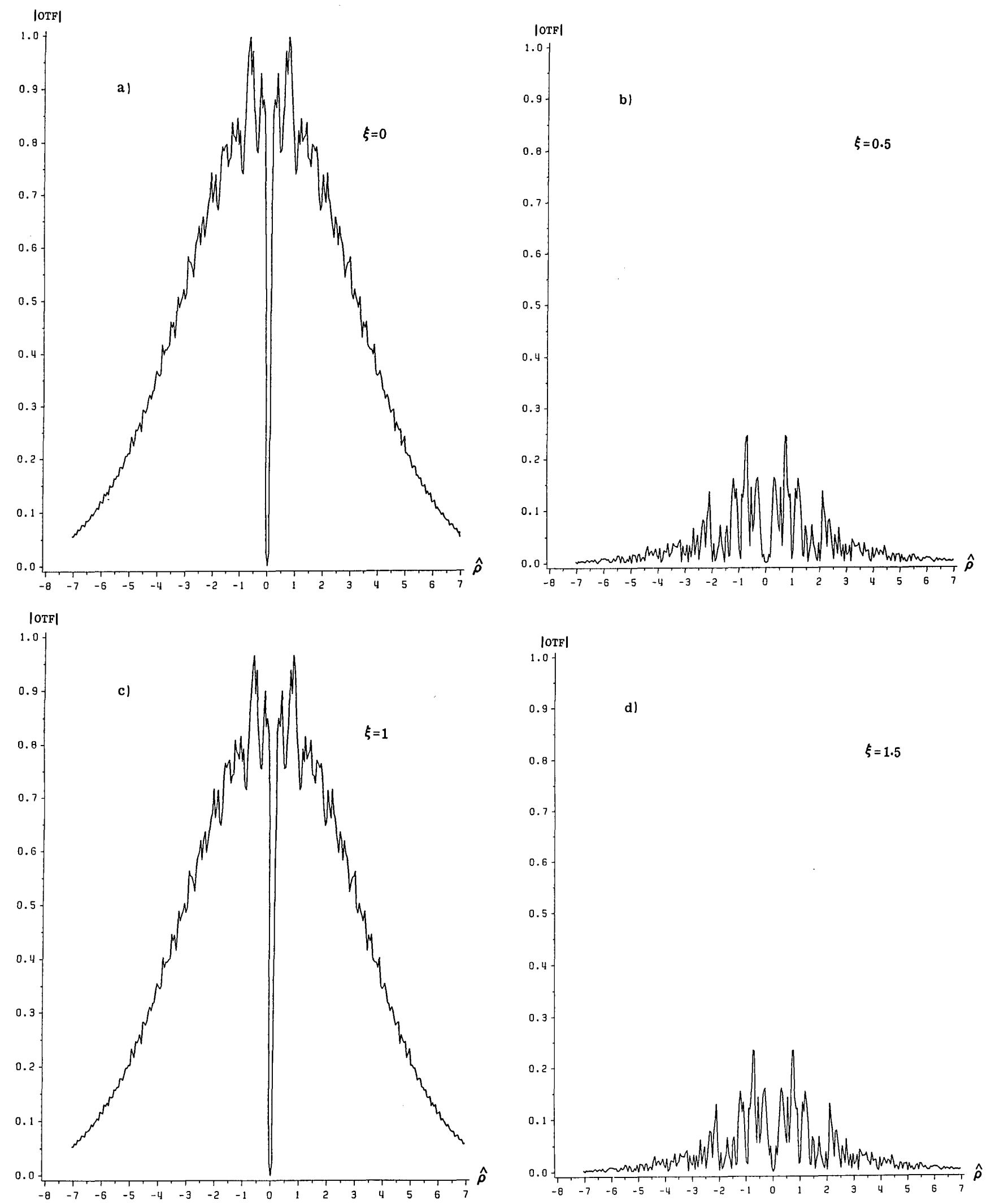

(Fig. 5 continued) 


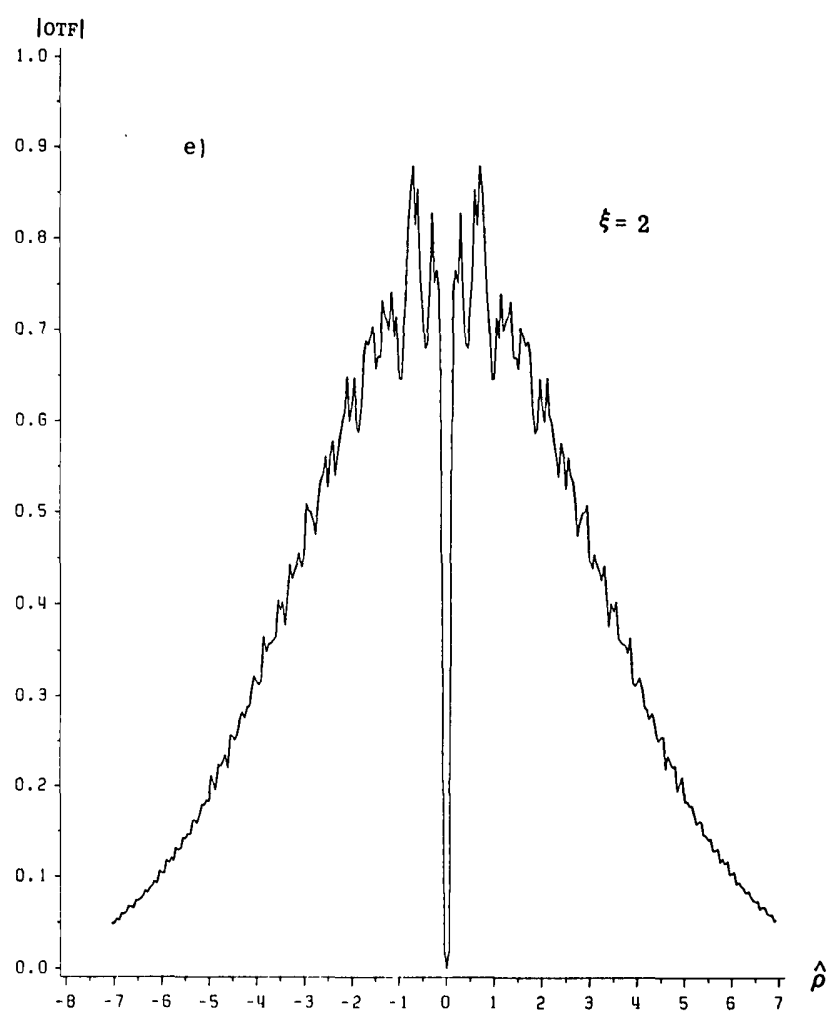

$$
\begin{aligned}
\operatorname{OTF}(\hat{\rho}, \xi)=\sum_{n} & \sum_{m} A_{n} A_{m}(-1)^{m} \pi \tilde{N} \\
& \times \exp \left(-\frac{\hat{\rho}^{2} \tilde{N}}{4}\left\{(\bar{N})^{-2}+[2 \pi(m+n+2 \xi)]^{2}\right\}\right),
\end{aligned}
$$

where

$$
\frac{1}{\tilde{N}}=\frac{2}{N}-j 2 \pi(n-m) \text {. }
$$

In what follows, we present some numerical results showing the evolution of the OTF given in Eq. (21) with the normalized defocus distance $\xi$. Two cases are shown. In the first case, we take $\alpha=\beta=0.5$, which corresponds to having complementary pupils. $N$ is taken to be 9 . Figure 4 shows the OTFs at various distances $\xi$. In the second case, $\alpha=\beta=0.1$, which is a good approximation to the thin ring pupils given by Eq. (12). Figure 5 shows the results. We indeed observe that the OTF is of bandpass characteristics and is periodic along $z$ with a periodic distance $z_{0}$.

\section{Conclusions and Remarks}

We have explored the possibility of realizing an incoherent spatial filtering which is repeated periodically along $z$. The condition for $z$-periodicity is that the domain of the two interacting pupils be limited to discrete Montgomery rings of radii proportional to the $\sqrt{n}(n=$ integer). We have shown that by employing Fresnel zone plates as the pupils in a two-pupil system, bandpass filtering with longitudinal periodicity can be achieved. Such periodic behavior of a spatial filtering
Fig. 5. Same as Fig. 4 but for $\alpha=\beta=0.1$ and $N=9$ : (a) $\xi=0$, (b) $\xi=0.5$, (c) $\xi=1$, (d) $\xi=1.5$, (e) $\xi=2$.

operation may have potential applications in 3-D image processing or coding. As a final note we want to point out that in Eq. (9) $\mu_{n}$ and $\nu_{m}$ can in general be a function of the azimuthal angle $\theta$. Rings with modulation along $\theta$ may give rise to other interesting or more general (nonrotationally symmetric) spatial filtering operations.

This material is based on work supported by the National Science Foundation under grant ECS8813115.

\section{References}

1. F. Talbot, "Facts Relating to Optical Science," Philos. Mag. 9, 401-407 (1836).

2. E. Lau, "Beugungsercheinungen an Doppelrastern," Ann. Phys. 6, 417 (1948).

3. W. D. Montgomery, "Self-Imaging Objects of Infinite Aperture," J. Opt. Soc. Am. 57, 772-778 (1967).

4. J. Jahns, A. W. Lohmann, and J. Ojeda-Castañeda, "Talbot and Lau Effects, a Parageometrical Approach," Opt. Acta 31, 313317 (1984).

5. G. Indebetouw, "Propagation of Spatially Periodic Wavefields," Opt. Acta 31, 531-539 (1984).

6. K. Patorski, "Talbot Interferometry with Increased Shear," Appl. Opt. 24, 4448-4453 (1985).

7. S. Jutamulia, T. W. Lin, and F. T. S. Yu, "Real-Time ColorCoding of Depth using a White-Light Talbot Interferometer," Opt. Commun. 58, 78-82 (1986).

8. C. Colautti, E. E. Sicre, and M. Garavaglia, "Incoherent Optical Spatial Filtering Based on the Lau Effect," Opt. Commun. 57, 16-20 (1986).

9. B. Packross, R. Eschbach, and O. Bryngdahl, "Image Synthesis Using Self-Imaging," Opt. Commun. 56, 394-398 (1986). 
10. N. Saiga and Y. Ichioka, "Visualization of the Strain Wave Front of a Progressive Acoustic Wave Based on the Talbot Effect," Appl. Opt. 24, 1459-1465 (1985).

11. J. Jahns and A. W. Lohmann, "OTF Synthesis: Low Pass and Band Pass Filters Realized by Periodic Replication of the Pupil Function," Opt. Commun. 42, 231 (1982).

12. D. Goerlitz and F. Lanzl, "Method of Zero-Order Non-Coherent Filtering," Opt. Commun. 20, 68 (1977).

13. A. W. Lohmann and W. T. Rhodes, "Two-Pupil Synthesis of Optical Transfer Functions," Appl. Opt. 17, 1141-1151 (1978).

14. P. Chavel and S. Lowenthal, "Implementation of an Incoherent Optical Image Restoration Method: Limitations Related to Optical Subtraction," Appl. Opt. 20, 1438-1449 (1981).

15. I. Glaser, "Information Processing with Spatially Incoherent Light," in Progress in Optics, Vol. 24, E. Wolf, Ed. (Publisher, Location, 1987), Chap. 5.

16. J. Jahns and A. W. Lohmann, "Opto-electronical Bandpass Filtering Applied to Incoherent Image Subtraction," Opt. Commun. 46, 85 (1983).

17. H. H. Hopkins, Wave Theory of Aberrations (Oxford U. P., London, 1950).
18. W. Stoner, "Incoherent Optical Processing via Spatially Offset Pupil Masks," Appl. Opt. 17, 2454-2467 (1978).

19. N. Konforti and E. Marom, "Two-Dimensional Optical Low Frequency De-Emphasis of the Modulation Transfer Function," Opt. Commun. 54, 212 (1985).

20. E. N. Leith and D. K. Angell, "Generalization of Some Incoherent Spatial Filtering Techniques," Appl. Opt. 25, 499-502 (1986).

21. W. T. Rhodes, "Bipolar Point Spread Function Synthesis by Phase Switching,” Appl. Opt. 16, 265-267 (1977).

22. T.-C. Poon and A. Korpel, "Optical Transfer Function of an Acousto-Optic Heterodyning Image Processor," Opt. Lett. 4, 317-319 (1979).

23. T.-C. Poon, "Scanning Holography and Two-Dimensional Image Processing by Acousto-Optic Two-Pupil Synthesis," J. Opt. Soc. Am. 2, 521-527 (1985).

24. G. Indebetouw and T.-C. Poon, "Parallel Synthesis of Biopolar Point Spread Functions in a Scanning Heterodyne Optical System," Opt. Acta 33, 827 (1986).

25. T.-C. Poon, J. Park, and G. Indebetouw, "Optical Realization of Textural Edge Extraction,” Opt. Commun. 65, 1 (1988). 